'Departamento de Ética del Colegio Médico de Chile A. G. ${ }^{2}$ Facultad de Medicina y Programa de Ética y Políticas Públicas en Reproducción Humana, Universidad Diego Portales.

${ }^{3}$ División de Obstetricia y

Ginecología, Escuela de Medicina, Facultad de Medicina, Pontificia Universidad Católica de Chile.

${ }^{4}$ Directora Médica, Clínica Hospital del Profesor. ${ }^{5}$ Departamento de Ciencias Neurológicas, Universidad de Chile.

6Programa de Genética Humana, ICBM, Facultad de Medicina, Universidad de Chile. ${ }^{7}$ Servicio de Medicina Interna, Hospital San Juan de Dios. Santiago de Chile. ${ }^{8}$ Departamento de Neuropediatría, Universidad de Valparaíso.

${ }^{9}$ Departamento de Bioética y Humanidades Médicas, Facultad de Medicina, Universidad de Chile.

10Primer Director del Programa Regional de Bioética OPS, Ex Ministro de Salud, Ex Presidente Departamento de Ética, Colegio Médico de Chile A. G.

${ }^{a}$ Abogado, Departamento Jurídico, Colegio Médico de Chile A.G.

Recibido el 6 de enero de 2016, aceptado el 12 de enero de 2016.

Correspondencia a: Dra. Sofía P. Salas

Ejército 260; Santiago, Chile. sofia.salas@udp.cl

\section{El médico y la objeción de conciencia. Opinión del Departamento de Ética del Colegio Médico de Chile A. G.}

\author{
SOFÍA P. SALAS ${ }^{1,2}$, MAURICIO BESIO $^{1,3}$, \\ GLADYS BÓRQUEZ ESTEFÓ ${ }^{1,4}$, RODRIGO A. SALINAS ${ }^{1,5}$, \\ CARLOS Y. VALENZUELA ${ }^{1,6}$, CONSTANZA MICOLICH ${ }^{1,7}$, \\ FERNANDO NOVOA SOTTA ${ }^{1,8}$, LIONIEL BERNIER VILLARROEL $^{1,9}$, \\ JULIO MONTT M. ${ }^{1,10}$, ADELIO MISSERONI RADDATZ ${ }^{1, a}$
}

\section{Position paper from the Department of Ethics of the Chilean College of Physicians about conscientious objection}

The Chilean bill that regulates abortion for three cases (Bulletin No 9895-11) includes the possibility that health professionals may manifest their conscientious objection (CO) to perform this procedure. Due to the broad impact that the issue of CO had, the Ethics Department of the Chilean College of Physicians considered important to review this concept and its ethical and legal basis, especially in the field of sexual and reproductive health. In the present document, we define the practical limits of $C O$, both for the proper fulfilment of the medical profession obligations, and for the due respect and non-discrimination that the professional objector deserves. We analyze the denial of some health institutions to perform abortions if it is legalized, and we end with recommendations adjusted to the Chilean reality. Specifically, we recognize the right to conscientious objection that all physicians who directly participate in a professional act have. But we also recognize that physicians have ineludible obligations towards their patients, including the obligation to inform about the existence of this service, how to access to it and-as set out in our code of ethics- to ensure that another colleague will continue attending the patient.

(Rev Med Chile 2016; 144: 382-387)

Key words: Abortion, induced; Ethics; Professionalism.
E 131 de enero de 2015, la Presidenta de Chile envió al parlamento un proyecto de ley que regula la despenalización de la interrupción voluntaria del embarazo en tres causales: cuando la mujer se encuentra en riesgo vital, cuando el embrión o feto padezca una alteración letal, y cuando el embarazo es resultado de una violación ${ }^{1}$. Dicho proyecto reconoce el derecho del médico a negarse a realizar el procedimiento si ha manifestado su condición de objetor de conciencia a las autoridades correspondientes y limita dicha libertad de conciencia "en el caso que la mujer requiera atención médica inmediata e impostergable", circunstancia en la cual no puede "excusarse de realizar la interrupción del embarazo en la medida que no exista otro (a) médico (a) cirujano (a) que pueda realizar la intervención"'.

Dada la importancia del tema de la objeción de conciencia (OC), el Departamento de Ética del Colegio Médico ha considerado necesario revisar este concepto y su fundamentación ética y legal, especialmente en el ámbito de la salud sexual y 
reproductiva. En este documento, definiremos los límites prácticos de la OC, tanto para el adecuado cumplimiento de la obligación que la profesión médica tiene con sus pacientes, como para el debido respeto y no discriminación hacia el profesional objetor; analizaremos la postura de algunas instituciones al negarse a realizar abortos si se legisla al respecto, y terminaremos con recomendaciones ajustadas a la realidad chilena.

\section{Concepto de objeción de conciencia (OC)}

La OC es un conflicto clásico entre el deber ante la ley y el deber ante la propia conciencia, por lo cual se permite el derecho a resistir los mandatos de la autoridad cuando éstos van en contra de los principios morales del individuo. La OC, la desobediencia civil, u otra acción de negación a seguir una ley, se dan ante situaciones críticas de conflictos valóricos irreductibles. Un ejemplo clásico es la oposición a realizar el servicio militar obligatorio por parte de ciudadanos que aducen razones de conciencia moral ${ }^{2}$.

En este documento, abordaremos el conflicto de la OC desde la perspectiva de los profesionales de la salud. La conciencia es un atributo del individuo, basada en un juicio moral reflexivo sobre lo que considera un bien o un mal, que trasciende la mera consciencia neurológica, abarcando la consciencia reflexiva y moral de ese ser humano, y que nos obliga a decidir respetando esos principios ${ }^{3}$. A su vez, entendemos por OC profesional a la negativa a cumplir un cometido profesional permitido por las leyes impuestas por las autoridades legítimas, aduciendo para ello razones morales o de conciencia. La Declaración Universal de Derechos Humanos señala que la libertad de conciencia y la de expresión son derechos humanos fundamentales, a los cuales se les garantiza máxima protección en la mayoría de las constituciones y tratados internacionales ${ }^{4}$. En este sentido, los derechos de la persona que requiere acceder a una prestación de salud y el derecho del profesional a respetar su propia conciencia, no pueden ser considerados simplemente un problema entre privados, sino que involucran a la sociedad entera, que debe encontrar una forma de armonizar el balance entre estos intereses en conflicto, sin perjudicar a ninguna de las partes.

La discusión acerca de la OC de los profesio- nales sanitarios nos remite al debate, más general, respecto de si es deseable que la sociedad excuse a uno de sus miembros de cumplir sus obligaciones legales, cuando éste posea arraigadas creencias por las que juzga que el cumplimiento de éstas es éticamente erróneo. Cabe señalar que el interés de la sociedad por respetar la OC está definido por la importancia que la comunidad le asigna al respeto de la integridad moral de los individuos que la componen y al derecho a conducir sus vidas de acuerdo a las propias convicciones morales. Esta integridad adquiere especial significado para quienes ejercen la medicina, que tienen asignadas responsabilidades que exigen un nivel de reflexión moral que se vería menoscabado si no se les diera la posibilidad de seguir los dictados de su propia conciencia ${ }^{5}$.

La OC es más bien excepcional, si consideramos a todo el cuerpo legal, porque los legisladores intentan plasmar en las leyes el respeto a las valoraciones de toda esa sociedad para la cual las leyes se preparan. Sin embargo, hay situaciones donde tal consenso valórico es imposible y esto da pie a varias posiciones de las personas discrepantes con la ley, tales como la OC, la desobediencia civil, y la rebelión, entre otras. Se distingue la OC de la desobediencia civil, sin embargo, en que aquélla no implica ningún llamado al sentido de justicia de la mayoría. No tiene el propósito político de denunciar una ley que cree injusta, sino sólo busca evitar que el objetor, al cumplirla, violente su propia conciencia. Por lo tanto, la OC no es un acto que ocurra en el "foro público", sino que es una acción de naturaleza estrictamente individual, cuyo carácter no secreto reside en que este último opera bajo la presunción que la autoridad está consciente de su rechazo a cumplir con determinadas leyes ${ }^{6}$.

\section{Alcances a la objeción de conciencia}

Para que una negativa a cumplir la ley satisfaga los requisitos que la sitúan dentro de la categoría de OC y pueda aspirar a ser aceptada como tal en el ordenamiento legal, no basta con que su fundamento se encuentre en una profunda convicción moral o religiosa. Debe cumplir, además, con ciertas condiciones formales que los tratadistas han reconocido como esenciales para reclamar esta categoría. Para John Rawls, por ejemplo, son 
características esenciales de la OC, -que comparte con la desobediencia civil- el que no se oculte la infracción a las autoridades, y que no utilice la fuerza ni vulnere los derechos de terceros en su expresión. En la esencia de ambas se encuentra la no-violencia ${ }^{6}$.

En el caso de la profesión médica, esta circunstancia debe tomar en cuenta, además, el compromiso tácito que los médicos tienen con la sociedad, de anteponer el bienestar de su paciente a cualquiera consideración de carácter personal. A la profesión médica la distingue un compromiso público con el bienestar de sus pacientes; este compromiso es el que sustenta la confianza que debe existir en la base de la relación médico-paciente, y que de encontrarse ausente, la desvirtuaría en su esencia ${ }^{7}$. El Manual de Ética Médica de la Asociación Médica Mundial, que nuestro Código de Ética reproduce, afirma que: "El profesionalismo médico no incluye solamente la relación entre un médico y su paciente, ...también implica una relación con la sociedad", de tal modo que en los cimientos de la profesión médica se encuentra el compromiso ético de servir los intereses de los pacientes por sobre aquellos de carácter estrictamente personal ${ }^{8}$. Existe, entonces, una aparente contradicción entre la comprensión del ejercicio de la medicina como una profesión y la voluntad de hacer prevalecer convicciones morales personales, por sobre eventuales derechos del paciente a acceder a prestaciones legítimas ${ }^{9}$. Esto es también motivo de debate, puesto que algunos médicos consideran que la OC no se aplica para beneficio personal -como sería el evitar dañar la propia conciencia- sino que estiman que no realizar el aborto va en el mejor beneficio del paciente, al considerar al embrión o feto también como paciente ${ }^{10}$.

En el campo de la salud sexual y reproductiva, el tema de la OC requiere de un enfoque novedoso por los derechos que están amenazados, puesto que se pueden afectar intereses relevantes de terceros, poniendo en riesgo la vida, la salud y la integridad física o la autonomía de las personas. Sin embargo, son precisamente en estas situaciones donde se amenaza la vida e integridad de los embriones y fetos, cuyos derechos no estarían siendo protegidos en el caso del aborto. Es la valoración de esos "terceros" y de sus derechos el motivo de choque valórico aparentemente irreductible. Como veremos más adelante, de las tres formas de resolver este conflicto -una posición absoluta por la cual el objetor no está obligado a derivar o informar, otra fundada en el profesionalismo irrestricto, según la cual los estándares de lo exigido por la sociedad son irrenunciables, por lo que no hay lugar para la OC; y otra intermedia, que busca conciliar los derechos de ambos- nosotros proponemos esta última.

\section{Fundamentos de la objeción de conciencia}

En una sociedad democrática y plural en lo valórico, debemos ser capaces de articular el espacio político y público con el espacio personal. Como mencionamos antes, la Declaración Universal de Derechos Humanos consagra el derecho que tiene toda persona "a la libertad de pensamiento, de conciencia y de religión", estableciendo implícitamente que existe el derecho a negarse a actuar en contra de sus propios valores y creencias y reconoce que la persona tiene el derecho a construir su propia escala de valores y de actuar en consecuencia ${ }^{4,11}$. Asimismo, la Constitución Política de Chile asegura a todas las personas "la libertad de conciencia, la manifestación de todas las creencias y el ejercicio libre de todos los cultos que no se opongan a la moral, a las buenas costumbres o al orden público"; de este derecho suele deducirse el derecho a la $\mathrm{OC}^{12}$.

\section{Límites de la objeción de conciencia}

La Declaración de Oslo de la Asociación Médica Mundial sobre el aborto terapéutico, señala que "(si) las convicciones del médico no le permiten aconsejar o practicar un aborto, éste puede retirarse, siempre que garantice que un colega calificado continuará prestando la atención médica" y agrega que es deber de la profesión médica asegurar tanto la protección de sus pacientes como el defender los derechos del médico en la sociedad ${ }^{13}$. A su vez, el Código de Ética del Colegio Médico de Chile acoge el valor de la conciencia al señalar que "el médico a quien se solicitaren prestaciones que vayan en contra de su conciencia o de su convencimiento clínico, podrá negarse a intervenir. En estas circunstancias, procurará que otro colega continúe asistiendo al paciente, salvo que ello produjere graves e inmediatos daños para la salud del enfermo" . Asimismo, el Grupo Interdiscipli- 
nario de Bioética del Instituto Borja de Bioética argumenta del siguiente modo: "La objeción de conciencia no es un derecho absoluto, sino que tiene sus límites en cuanto que puede vulnerar derechos de otras personas. Por ello, debe hacerse un ejercicio coherente y responsable, explicitando la condición de objetor y los límites de la misma, con el fin de permitir una correcta organización de las prestaciones, evitando el perjuicio de terceros" 14 . También ha adoptado esta postura la Asociación Médica Mundial; en su documento sobre Profesionalismo Médico, reconoce que los médicos tienen derecho a permanecer leales a sus valores y moral personales y a ejercer su juicio profesional independiente, y que poseen el derecho a informar a sus pacientes de aquello, pero no de un modo que sea indebidamente coercitivo o argumentativo. Señala explícitamente que debe hacerse distinción entre una situación aguda, donde se requiere de cuidado inmediato para mantener la salud, en contraposición a una situación menos aguda en la que se dispone de tiempo para que otro facultativo realice la prestación. Asimismo, señala que los médicos deben entregar a sus pacientes toda la información que requieran, independientemente de sus propios valores, contribuyendo así a que los pacientes puedan dar un consentimiento informado válido. Este concepto es uno de los pilares centrales de la ética médica moderna y no puede verse socavado por la $\mathrm{OC}^{13}$.

La OC se enmarca así en un nuevo tipo de relación clínica que reconoce que el profesional es un ciudadano con valores personales, pero también con obligaciones profesionales, éticas y jurídicas definidas colectivamente, de tal modo que debe procurar no desatender a sus pacientes, tal como lo señala nuestro Código de Ética ${ }^{8}$. Esta obligatoriedad, en el sentido de procurar que otro colega continúe asistiendo al paciente en aquellos casos en que la atención sea negada por razones de conciencia, es congruente con las obligaciones deontológicas contenidas en otras normativas, como por ejemplo los casos de Francia y Gran Bretaña ${ }^{15,16}$. Especial cuidado hemos tenido en el alcance de la redacción de estos artículos en los códigos citados. Es fácil darse cuenta que en todos ellos existe una cautela respecto a lo que se entiende por "derivar", "procurar" y en qué consiste la atención médica que se busca procurar al derivar a otro profesional. Existe para nosotros una explicación para este hecho y se debe a las distintas posturas que existen en relación a la licitud ética de los procedimientos sujetos de objeción de conciencia, diferencias presentes también en nuestra agrupación. Esta cautela y cuidado se mantienen en las conclusiones de este documento. En este sentido, el profesional asume una obligación de no abandono de su paciente; es decir, no puede simplemente retirarse de la atención aduciendo OC, sino que debe asegurar que otro proveedor continúe con la atención. A su vez, este reconocimiento al derecho a la OC se ha restringido a aquellas personas que directamente participan de la acción y no a funcionarios administrativos ni mucho menos a aquellos que deben otorgar asistencia sanitaria a una mujer que ya se hizo un aborto y que sufre las complicaciones del mismo $^{11,17}$.

\section{Negativas institucionales a realizar una acción de salud legal}

Como ya hemos mencionado, la OC debe ser entendida como un derecho personal, basado en las creencias más profundas del individuo, cuyo fundamento ético es la libertad ideológica, la cual es atributo de las personas. Respecto de la OC de las instituciones de salud, es evidente que las instituciones no son poseedoras de conciencia, aunque es legítimo reconocer que existen instituciones privadas con idearios valóricos y códigos de ética institucionales que guían y orientan su trabajo, y que en ese contexto no entregan ciertas prestaciones a una población debidamente informada y que por tanto, comparte esos mismos valores. En el caso de un sistema de atención cerrado, donde la persona que requiere la prestación no tiene posibilidad de elección, se hace necesario que exista una organización adecuada para que las personas puedan trasladarse fácilmente a otras instituciones donde puedan ser atendidas de manera oportuna ${ }^{11}$. La institución pública, en cambio, no puede negarse a entregar una prestación legalmente establecida, puesto que actúa en representación del Estado, debiendo procurar que dentro de su equipo de salud existan funcionarios no objetores. Asimismo, sería inadmisible que una autoridad de un centro de salud, en base a sus creencias o valores personales, imponga normas en la institución que tiene a su cargo, que limiten los derechos de terceros a obtener prestaciones sanitarias legales. 


\section{Conclusiones y recomendaciones}

La OC es un problema inevitable en una sociedad pluralista y libre, pero requiere ser regulado. Por un lado, la decisión del objetor de oponerse al poder del Estado cuando ve en riesgo sus principios más profundos muestra el valor de la integridad y convicción moral y la sociedad debe resguardar dicho valor. Por otro lado, el Estado no puede desatender el derecho que tienen las personas de acceder a legítimas prestaciones de salud. Por lo tanto, para que esa OC sea verdadera, nos parece necesaria la regulación de ciertos aspectos específicos:

¿Quiénes pueden ser objetores? Consideramos que este derecho le asiste sólo a aquellos que realizan el acto clínico directo, sea terapéutico o quirúrgico, pero no a aquellos que tratan sus complicaciones o posibles consecuencias, ni tampoco quienes participan del cuidado posterior de la persona que se ha realizado el procedimiento.

¿Se debe crear un Registro de objetores? Pensamos que el registro de la declaración del objetor no debe ser público, para no ser sujeto de discriminación, pero si debe ser conocido por las autoridades de la institución que lo tiene contratado. Forma parte de la prudencia que acompaña el ejercicio de nuestra profesión, el evitar exponerse a situaciones en que el objetor es el único prestador, especialmente cuando es posible prever la inexistencia de una posibilidad real de derivación del paciente.

En resumen, la necesidad de reconocer y regular la objeción de conciencia de los profesionales, necesita de la existencia previa de una ley que asegure acceso universal, sencillo y no discriminatorio de las prestaciones de salud, tanto en el sistema de salud público como en el privado. Para que se respeten los derechos de las personas que requieren de la prestación y también el de los objetores, éstos deben manifestar previamente y de manera fundamentada, su condición de objetor de conciencia a realizar determinadas prestaciones de salud ante el director o jefe de servicio, quien debe estar en conocimiento respecto de si cuenta o no con personal capacitado que esté dispuesto a otorgar la prestación. Éste, a su vez, debe realizar las acciones necesarias para que la población de usuarios no sólo no quede desatendida, sino que reciban aquella prestación que requieren, puesto que las instituciones sanitarias de carácter público deben otorgar las prestaciones legalmente reco- nocidas a todos los ciudadanos que las solicitan o proveer de las instancias de derivación correspondientes.

Por último, es necesario enfatizar que es deber del Estado organizar la atención de manera tal que se oferte a la población los especialistas suficientes a lo largo del país.

\section{Acuerdo de conclusiones}

a. Tanto la objeción de conciencia, como el derecho de un usuario a acceder a una prestación, son valores que deben ser resguardados.

b. El objetor de conciencia tiene derecho a objetar su participación en la prestación, pero no a obstruirla.

c. El aseguramiento del acceso a la prestación es responsabilidad del Estado y no del objetor.

d. Todo médico que participa en una prestación profesional puede ejercer su derecho a objetar, independientemente del papel que le corresponda desempeñar en aquella, y sobre él recaen, asimismo, las obligaciones que más abajo se detallan.

e. El objetor tiene la obligación de informar sobre la existencia de la prestación y la manera de acceder a ella y -según se establece en nuestro Código de Ética- procurar que otro colega siga asistiendo al paciente.

f. El objetor no puede ser obligado a participar en la concreción de la prestación, excepto en aquellos casos en que la objeción de conciencia "produjere graves e inmediatos daños para la salud del enfermo", según se establece en el mismo código.

\section{Referencias}

1. Proyecto de ley que Regula la despenalización de la interrupción voluntaria del embarazo en tres causales. Boletín No 9895-11 del 31 de enero de 2015. Disponible en https://www.camara.cl/pley/pley_detalle.aspx?pr$\mathrm{mID}=10315$ \&prmBoletin=9895-11 (Consultado el 7 de julio de 2015).

2. Legal Information Institute Cornell University Law School. Cassius Marsellus CLAY, Jr. also known as Muhammad Ali, Petitioner, v. UNITED STATES. No. 783. En 403 U.S. 698 (91 S.Ct. 2068, 29 L.Ed.2d 810) 1971. Disponible en https://www.law.cornell.edu/supreme- 
court/text/403/698 (Consultado el 3 de septiembre de 2015).

3. Diccionario de la Real Academia Española. Disponible en http://lema.rae.es/drae/?val=conciencia (Consultado el 3 de septiembre de 2015).

4. Declaración Universal de los Derechos Humanos, 1948. Disponible en http://www.un.org/es/documents/udhr/ (Consultado el 3 de septiembre de 2015).

5. Wester G. Conscientious Objection by Health Care Professionals. Philosophy Compass 2015; 10 (7): 42737.

6. Rawls J. A theory of justice. Oxford University Press. 1999, Oxford, Reino Unido.

7. Pellegrino ED. Professionalism, profession and the virtues of the good physician. Mt Sinai J Med 2002; 69 (6): 378-84.

8. Colegio Médico de Chile AG. Código de Ética 2011. Ediciones Tierra Mía Ltda. Santiago de Chile.

9. Odell J, Abhyankar R, Malcolm A, Rua A. Conscientious objection in the healing professions: A reader's guide to the ethical and social issues. Abortion and Contraception, 2014. Disponible en http://hdl.handle. net/1805/3844 (Consultado el 3 de septiembre de 2015).

10. Besio M. Objeción de conciencia, Profesión médica y Proyecto sobre despenalización del aborto en Chile. Rev Med Chile 2016 (aceptado para su publicación).

11. Beca JP, Astete CA. Objeción de conciencia en la prácti- ca médica. Rev Med Chile 2015; 143 (4): 493-8.

12. Constitución Política de la República de Chile. Capítulo III, Art. 19: De Los Derechos y Deberes Constitucionales. Disponible en www.senado.cl/capitulo-iii-de-los-derechos-y-deberes-constitucionales/ prontus_senado/2012-01-16/093413.html (Consultado el 17 de noviembre de 2015).

13. Declaración de Oslo de la AMM sobre el Aborto Terapéutico. Adoptada por la 24a Asamblea Médica Mundial en Oslo, Noruega, agosto de 1970 y enmendada por la 35a Asamblea Médica Mundial en Venecia, Italia, octubre de 1983 y la 57a Asamblea General de la AMM, Pilanesberg, Sudáfrica, octubre de 2006. Disponible en www.wma.net/es/30publications/10policies/a1/ (Consultado el 17 de noviembre de 2015.

14. Busquets EA, Camacho JA, Cambra JF, Carrera J, Cusí V, Ferrer P, et al. Consideraciones sobre la objeción de conciencia. Bioètica \& debat 2012; 18: 3-19.

15. General Medical Council. Personal beliefs and medical practice. Conscientious objection. En: Good medical practice, 2013. Londres, Reino Unido. Disponible en http://www.gmc-uk.org/guidance/ethical_guidance/21177.asp (Consultado el 17 de noviembre de 2015).

16. Dickens BM, Objeción de conciencia y compromiso de conciencia. En: Bioética, reproducción y familia. F. Zegers and S. Salas, Editores. 2014, Universidad Diego Portales: Santiago de Chile. p. 146-82. 